\title{
Demystification of the Self-Seeded WDM Access
}

\author{
Anaëlle Maho, Gaël Simon, Sophie Barbet, François Lelarge, Fabienne Saliou, Philippe Chanclou, Paola Parolari, \\ Lucia Marazzi, Marco Brunero, Mario Martinelli, Fellow, OSA, Simon Arega Grebewold, \\ Juerg Leuthold, Fellow, OSA, and Romain Brenot
}

\begin{abstract}
The self-seeded cavity appeared in the last few years as a colorless and low cost solution for wavelength division multiplexing access. Although the self-seeded source presents a simple architecture, its behavior has been misunderstood for a long time. In this paper, we explain its operating principles and why we can define such a source as a laser. We evidence a laser threshold and show cavity modes for various lengths. We describe the conditions required by the reflective semiconductor optical amplifier to sustain the self-seeded cavity, by evaluating the choice of its epitaxial structure and the influence of its optical confinement factor. An analysis of the cavity behavior is given, pointing out that the relative intensity noise results from the beating noise between the cavity modes. An overview over the last performances in the $C$ - as well as in the O-band is then presented. Some practical applications are reported. In particular, we detail the mobile front-haul as a possible employment for the self-seeded cavity to achieve a self-organized wavelength network.
\end{abstract}

Index Terms-Colorless, reflective semiconductor optical amplifier (RSOA), self-seeded cavity, WDM PON.

\section{INTRODUCTION}

$\mathbf{S}$ INCE the principle of a self-seeded source has been proposed [1] many questions have arisen concerning its properties, working principle, and possible use in wavelength division multiplexing (WDM) networks. Even in the static regime, the nature of the emitted light remains unclear. The dynamic regime is even more unusual, since the round-trip time of photons in the cavity is much longer than the bit duration. Still, many groups have succeeded in modulating such a source at a few $\mathrm{Gb} / \mathrm{s}$. Most demonstrations have been made at $1.25 \mathrm{~Gb} / \mathrm{s}$ [2]-[5], while the use of fast reflective semiconductor optical amplifiers (RSOA)

Manuscript received May 31, 2015; revised July 29, 2015; accepted July 29, 2015. Date of publication August 6, 2015; date of current version February 5, 2016. This work was supported in part by the European Union's Seventh Framework Program (FP7/2007-2013) under Grant agreement ERMES no. 2885422012 and by the French National Research project LAMPION (Grant ANR-13-INFR-0002). A. Maho and G. Simon contributed equally to this work.

A. Maho, S. Barbet, F. Lelarge, and R. Brenot are with the III-V Labs, Route de Nozay, Marcoussis 91461 Cedex, France (e-mail: anaelle.maho@3-5lab. fr; sophie.barbet@3-5lab.fr; francois.lelarge@3-5lab.fr; romain.brenot@35lab.fr).

F. Saliou, G. Simon and P. Chanclou are with the Orange Labs, Lannion 22300, France (e-mail: fabienne.saliou@orange.com; gael.simon@ orange.com; philippe.chanclou@orange.com).

P. Parolari, L. Marazzi, M. Brunero, and M. Martinelli are with the Politecnico di Milano, Dip. Elettronica Informazione e Bioingegneria, Milano 20133, Italy (e-mail: paola.parolari@polimi.it; lucia.marazzi@ polimi.it; mario.martinelli@polimi.it).

S. Gebrewold and J. Leuthold are with the ETH University, Zürich 8092, Switzerland (e-mail: sgebrewold@ethz.ch; leuthold@ieee.org).
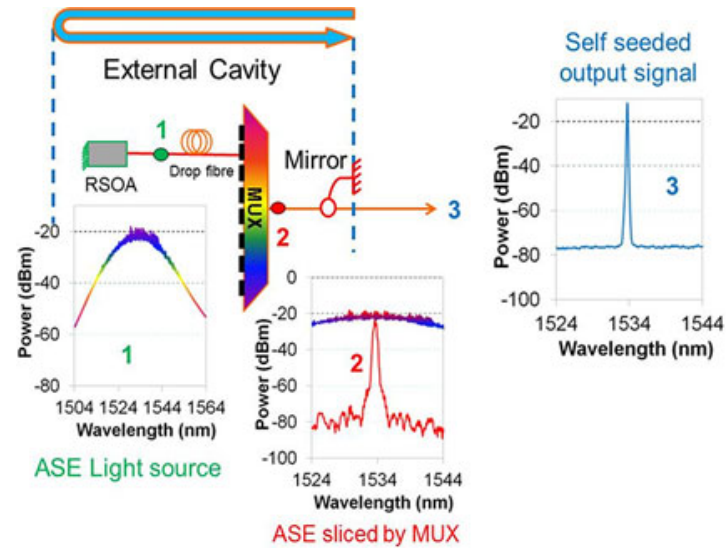

Fig. 1. A typical self-seeded source [11].

has even led to $10 \mathrm{~Gb} / \mathrm{s}$ demonstrations, both in the C-band [6] and O-band [7]. A self-seeded source usually exploits a RSOA, but it can also make use of Fabry-Perot lasers to reduce the temperature sensitivity and the spectral linewidth [8]. It should be mentioned that polarization sensitivity is no longer an issue since the use of Faraday rotators and mirrors compensates for the birefringence of the cavity elements [9]. We have recently managed to model the cavity [10], and fast gain compression in the gain medium has been identified as the key phenomenon enabling fast modulation. Some practical implementations have even been proposed for such a source, either for mobile fronthaul [11] or WDM overlay for access networks [4]. In this paper, we will shortly describe the source, and explain why it can be called a laser. We will then demonstrate how fast modulation is achieved. Finally, after describing the main properties of the built-in laser, we will summarize some practical use-cases in WDM networks.

\section{IS IT A LASER?}

\section{A. Principle of a Self-Seeded Cavity}

A self-seeded cavity is simply composed of a RSOA, a drop fiber, an arrayed waveguide grating (AWG) and a partial mirror. As shown in Fig. 1, a wide band amplified spontaneous emission (ASE) is emitted by the RSOA (point 1) and then filtered by the AWG (point 2). Part of the light is sent to the mirror and returns into the RSOA leading to a resonant cavity. This round trip occurs a certain amount of times before reaching the steady state. The other part of the light is hence sent to the network through a feeder fiber (point 3). 


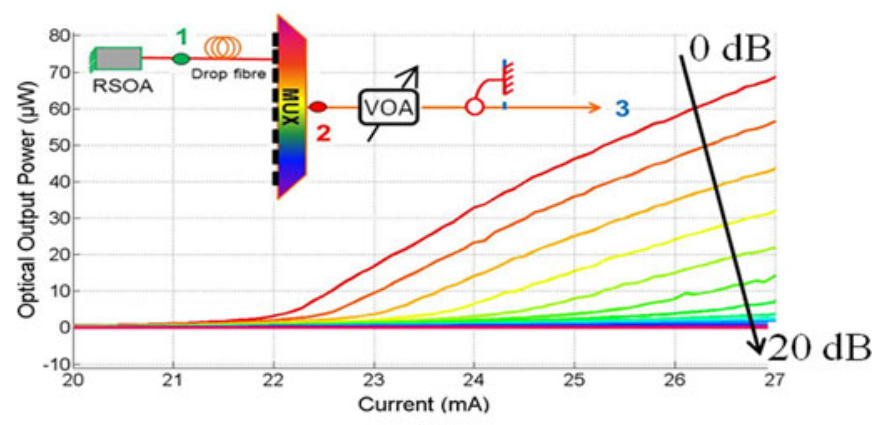

(a)

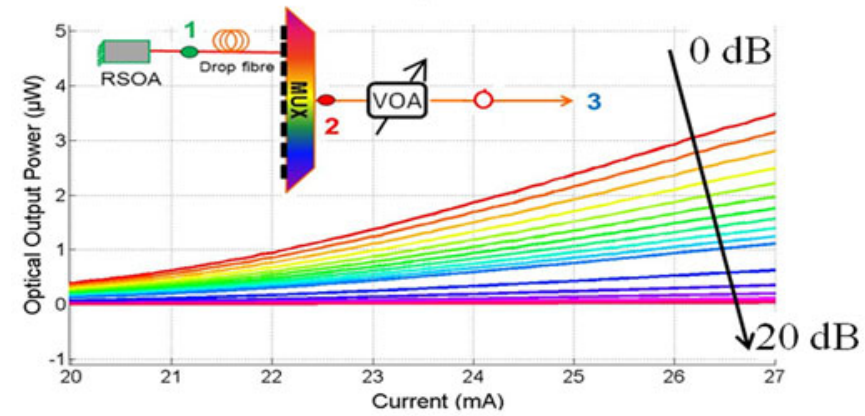

(b)

Fig. 2. Light-current curves with (a) and without (b) the mirror for various optical losses between the AWG and the splitter.

The emission wavelength is only determined by the selected AWG port so that every network terminal can be equipped with the same RSOA. Thus, this solution is passive and colorless.

\section{B. Static Behavior}

By definition, a laser is a device emitting light from a cavity where stimulated emission generates an optical gain which compensates the cavity losses. Based on this statement, we inserted a variable optical attenuator (VOA) between the AWG and the mirror (see Fig. 2(a) inset). Fig. 2(a) presents the output power versus the current injected into the RSOA for several optical attenuations. At $0 \mathrm{~dB}$, the threshold is well defined and becomes smoother when the attenuation increases. If we remove one mirror, opening the cavity, we eliminate the resonance and no threshold can be seen (see Fig. 2(b)).

More precisely, we measured the output power of these configurations at lower and higher bias currents than the threshold current (see Fig. 3). At low currents there is mainly spontaneous emission inside the RSOA, its gain is very low and cannot compensate the losses inside the cavity. In Fig. 3, the self-seeded source behaves like the open cavity. At higher currents, the stimulated emission dominates in the RSOA. Its output power is higher and increases the output power of the cavity. In particular, at $0 \mathrm{~dB}$, the power of the closed cavity is much higher than the power of the open one. For attenuations above $12 \mathrm{~dB}$, the gain is not sufficient to overcome the cavity losses. The self-seeded source presents a trend similar to the one of the open cavity. This behavior evidences the presence of a laser threshold.

Besides, we observed the output spectra for different cavity lengths (see Fig. 4). The measurements evidence the presence

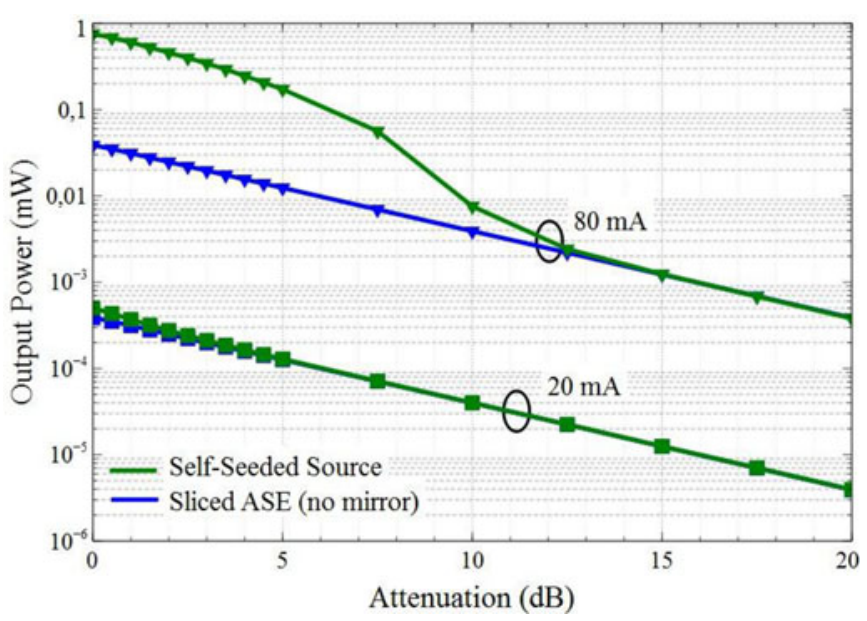

Fig. 3. Output power versus optical attenuation before (20 mA, squares) and after ( $80 \mathrm{~mA}$, triangles) the threshold current of a resonant (green) and an open (blue) cavity.
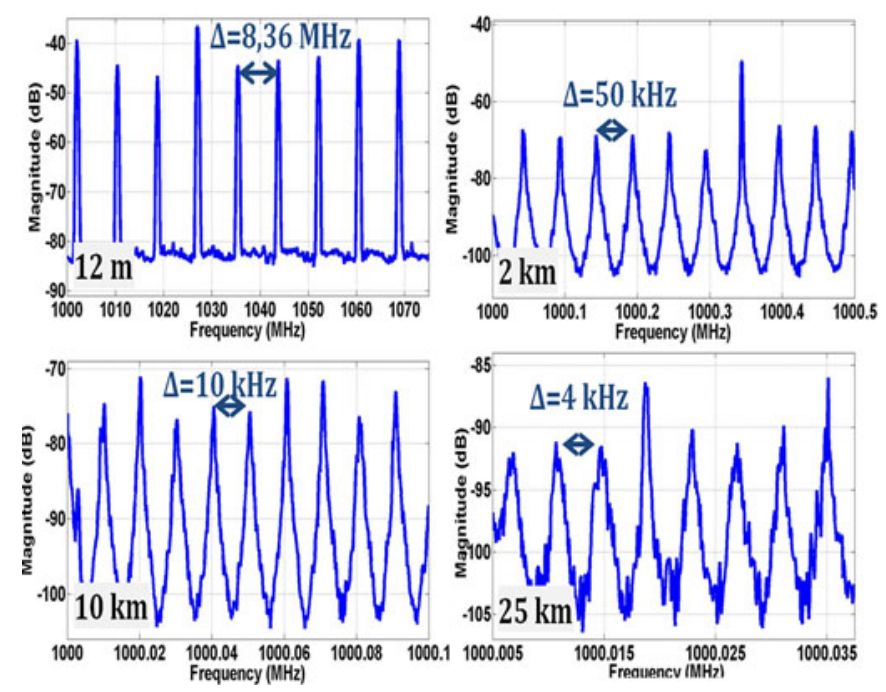

Fig. 4. Electrical spectra for optical cavities from $12 \mathrm{~m}$ to $25 \mathrm{~km}$.

of cavity modes whose spacing ranges from $8.36 \mathrm{MHz}$ to $4 \mathrm{kHz}$ for lengths from $12 \mathrm{~m}$ to $25 \mathrm{~km}$. These values match with the free spectral range (FSR) calculated with the length and the refractive index of the drop fiber length (see Fig. 5). As expected, the number of longitudinal modes in the cavity increases with its length.

Based on these considerations, we can consider the selfseeded source as a highly multimode laser with a low degree of coherence.

\section{HOW DOES IT WORK?}

\section{A. RSOA Requirements}

The RSOA is the key element of the self-seeded architecture. It has to provide enough gain to overcome the cavity losses and also to present a wide electro-optical bandwidth in order 


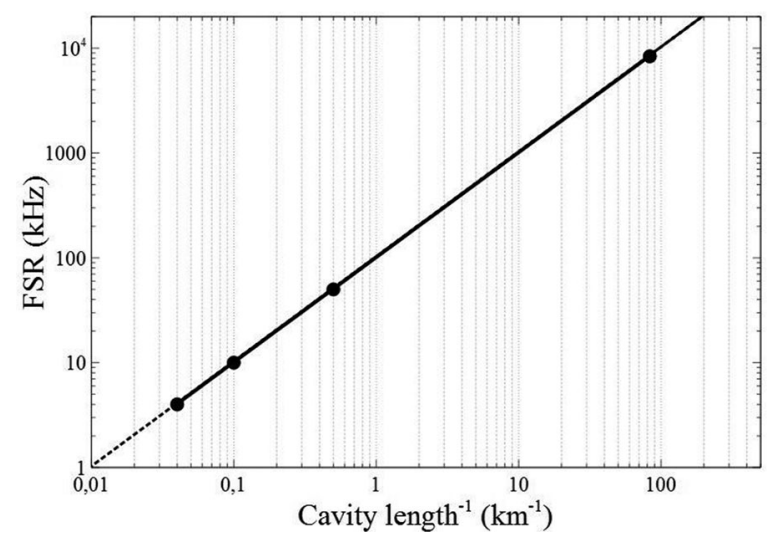

Fig. 5. Measured FSR versus the inverse of the cavity length.

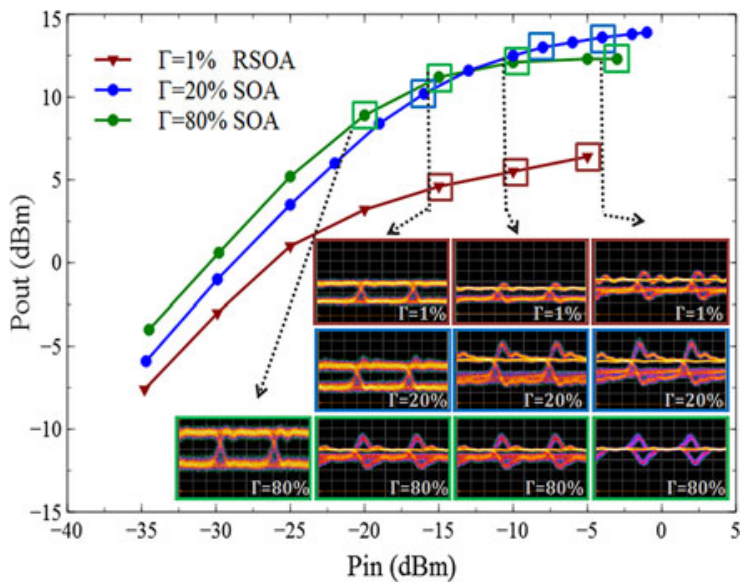

Fig. 6. Signal cancellation effect for optical confinement (Г) of $1 \%, 20 \%$ and $80 \%$. In the insets, erased eye diagrams at $5 \mathrm{~Gb} / \mathrm{s}$.

to be directly modulated. Nevertheless, these conditions are not sufficient to ensure a proper functioning.

Contrary to standard lasers, the round trip time in a selfseeded cavity frequently exceeds several tens of microseconds. Yet, at $10 \mathrm{~Gb} / \mathrm{s}$ the bit duration is $100 \mathrm{ps}$. Hence, at a given time the optical signals returning and outgoing from the cavity are not directly related to the injected current. To avoid interference with the new modulated signal, it is necessary to erase the returning data. This is obtained through gain compression [12]. Because of the saturation effect, the gain remains constant over a certain range of the input power. As shown in Fig. 6, for input power higher than $-10 \mathrm{dBm}$, the output power is approximately the same and the cancellation occurs. At high optical confinement $(\Gamma)$, there is a higher amount of ASE which saturates more the RSOA. Thus, the component is able to erase the returning signal more effectively and with a higher frequency response. However, high-optical confinement devices present a slower electro-optical response (see Fig. 7). We found that multiquantum wells (MQW) structures can be more appropriate as they can be directly modulated up to $10 \mathrm{~Gb} / \mathrm{s}$, while efficiently erasing returning signals.

Taking into account all of these considerations, we designed RSOAs in a compressively strained InGaAsP MQW material both in C- and O-band.

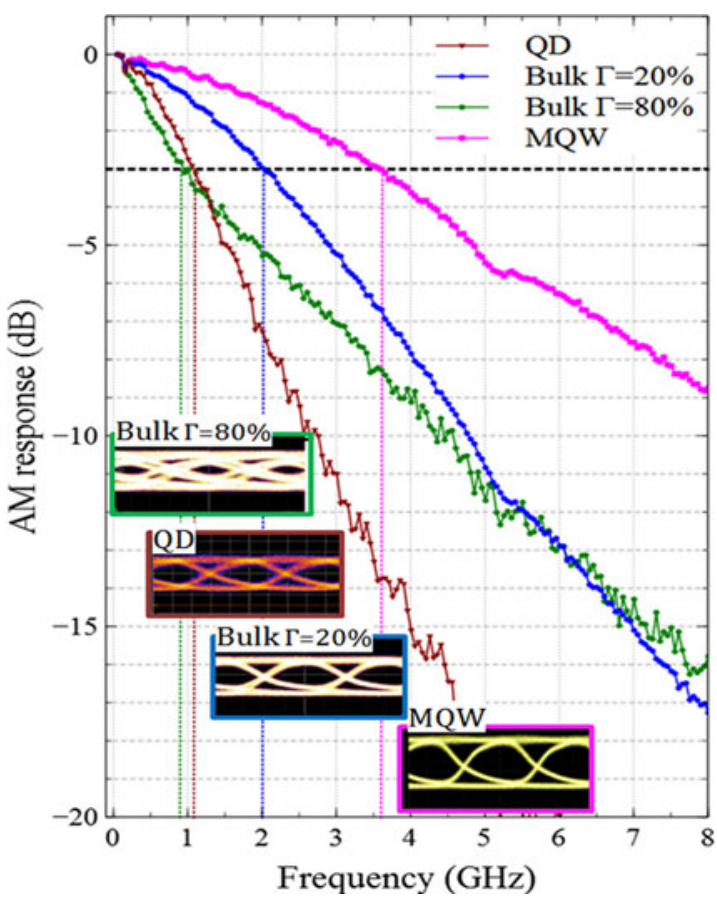

Fig. 7. Measured electro-optical bandwidths and eye diagrams at $5 \mathrm{~Gb} / \mathrm{s}$ for various RSOAs (direct ASE small-signal modulation at $100 \mathrm{~mA}$ ).

\section{B. Cavity Analysis}

Each element of the cavity impacts the performance. For instance, the birefringence of the drop fiber induces polarization variations inside the self-seeded cavity. Therefore the polarization control represents a key issue. In the early architectures [1] a fiber mirror associated with a low polarization dependent gain (PDG) RSOA was used to maximize the seeding efficiency, but the cavity was still sensitive to the polarization fluctuations over the time [2]. By replacing the fiber mirror by a Faraday rotator mirror and adding a Faraday rotator just after the RSOA, Martinelli et al. [9] obtained a polarization retracing circuit, which allowed the use of a high PDG RSOA.

Another important matter is the choice of the coupling ratio of the optical coupler which influences both the source output power and the transmitted signal quality. Increasing the optical power reflected back to the RSOA reduces the transmitted power and thus the optical budget [18]. On the other hand it enhances data modulation (see Fig. 6) and improves the receiver sensitivity. Depending on the configuration of the cavity, a tradeoff has to be found [14], [24].

As already evidenced the AWG determines the source emission wavelength. It also influences the quality of the transmitted signal. A narrow full width at half maximum (FHWM) increases the relative intensity noise (RIN) of the source, whereas a wider filter results in a larger output spectrum, which is more sensitive to chromatic dispersion impairments during the propagation [13], [17]. Moreover the bandwidth of the signal is not only determined by the AWG FHWM but also by its shape. The signal from a Gaussian filter is indeed wider than a signal from a flat-top filter [14] and will be more affected by the chromatic dispersion 
for the same FWHM. In both cases the laser wavelength is red shifted compared to the central wavelength of the AWG channel due to the Bogatov effect. For a flat-top shape the peak wavelength is close to the cut-off wavelength of the pass band [14]. Thus the AWG at the receiver side, the demultiplexer, filters useful data [16] which affects the transmission performance. To avoid such impairments the Faraday rotator mirror could be placed at the receiver end, after the demultiplexer, so that the signal shape is built by the cascade of the two AWGs. This implies to put the mirror at the optical line termination side, extending the cavity length which is then given by the drop and the feeder fibers. This choice increases the cavity losses, the roundtrip time and thus the time to reach the steady state [18]. The cavity build-up is relatively long: in reference [15], for a $420 \mathrm{~m}$ long cavity the output power reaches its steady state after six roundtrips whereas the optical spectrum and the bit error rate reach their steady state after more than 100 roundtrips. In this case, it takes almost $1 \mathrm{~ms}$ to complete the steady state in terms of power, bit error rate, and spectrum.

Due to the high number of longitudinal modes present in the long self-seeded sources, the measured RIN is quite large [19], [25]. A short cavity (a few meters) comprises hundreds of longitudinal modes and this number increases with the length, up to hundreds of thousands for km-long cavities. Fig. 8 presents the RIN measured for a 12-m long cavity: the beating noise peaks are spaced of the cavity FSR. As demonstrated by simulations from an accurate model [10], the competition between all the modes determines the RIN.

\section{How TO USE IT?}

Since the first publication in 2007 [1] many self-seeded architectures have been demonstrated. In Fig. 9 we present a comparison among some of the literature results. We focus on the achieved bit rate and the total distance between the RSOA and the receiver. Most of the realizations were performed in the Cband at $1.25 \mathrm{~Gb} / \mathrm{s}$ on transmission distances around $20 \mathrm{~km}$. In particular, a prototype [20] and a field trial with standard frames [11] reached these values. The coexistence of time division multiplexing and WDM PON supporting 512 users was studied in [4]. Although the burst mode is still limited by the build-up time of the long-laser, the rise time is compatible with a sleep mode for energy saving [18]. Performances in the C-band reach up to $5 \mathrm{~Gb} / \mathrm{s}$ over $25 \mathrm{~km}$ [19]. The main limitation factor is the chromatic dispersion [6], [15]. That is why O-band has been investigated [7], [16]. Long distances, over $70 \mathrm{~km}$, and high bit-rates, as $10 \mathrm{~Gb} / \mathrm{s}$, were obtained [7], [24]. In the absence of chromatic dispersion, the transmission is mainly limited by the cavity losses and the RIN.

Generally the RSOA based architectures present better performances than the alternative solutions employing Fabry-Perot lasers [8].

Depending on the application, there is a tradeoff to be chosen between the cavity length and the transmission distance. Cavity losses increase indeed with the distance, reducing the output power and thus the optical budget available for transmission. On the other hand, increasing the distance between the optical

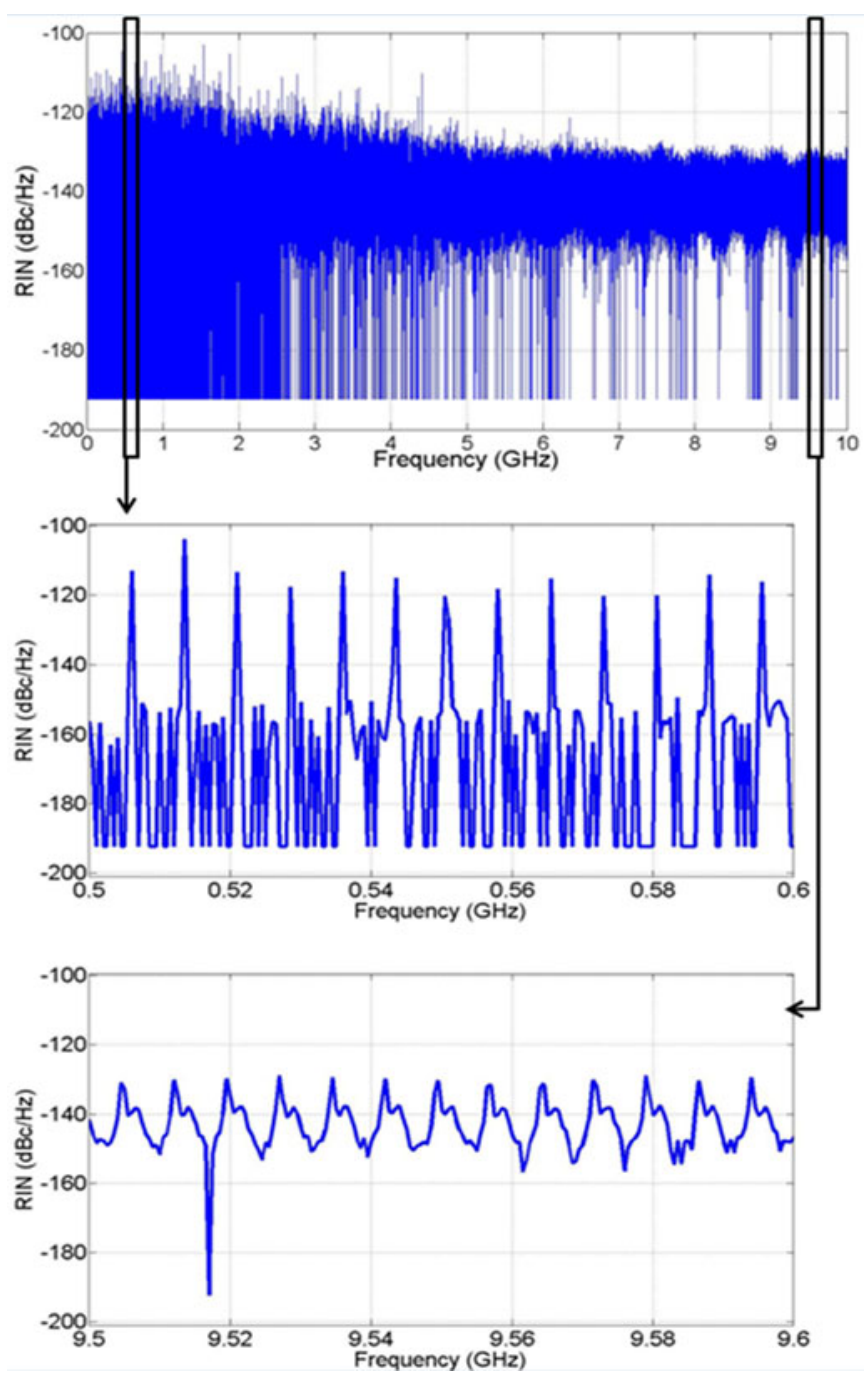

Fig. 8. Measured RIN of the 12-m long cavity.

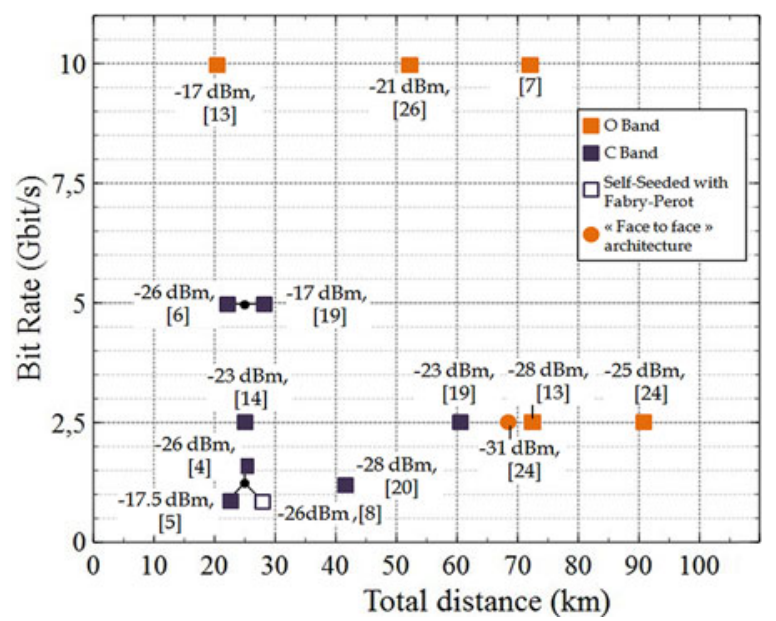

Fig. 9. Overview of bit rates and total distance (cavity length and transmission distance) presented in the literature for $\mathrm{C}$ - and $\mathrm{O}$ - band. Full square: standard self-seeded cavity, blank square: self-seeded cavity with Fabry-Perot, circle: face to face configuration. The receiver sensitivity is given for a BER of $10^{-3}$. 


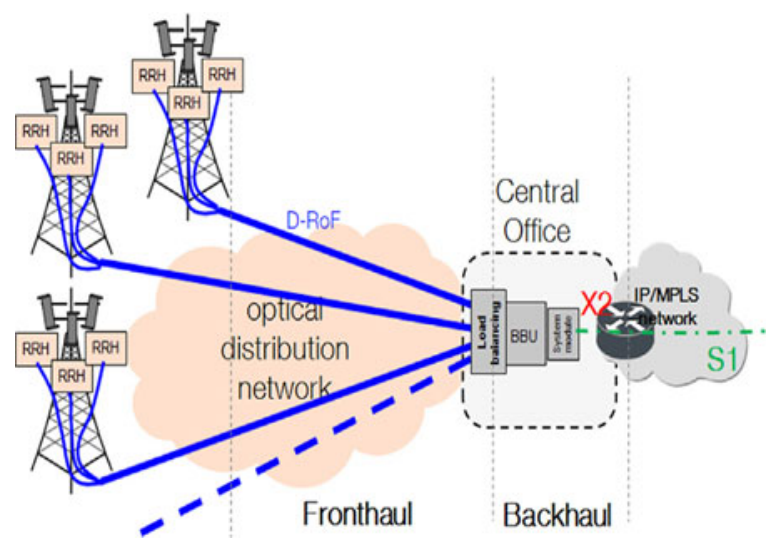

Fig. 10. Example of a mobile front-haul [21].

network unit (ONU) and the shared point enables to aggregate more users at the same node.

The self-seeded architecture appears to be suitable for the mobile front-haul. The upstream radio signal is first received by the antenna, processed by the remote radio head (RRH) close to the antenna and then sent to a base band unit (BBU) located in a base station which centralizes the data and communicates with the central office $(\mathrm{CO})$. The usual distance between the RRH and the $\mathrm{BBU}$ is $15 \mathrm{~km}$ with a maximum of $40 \mathrm{~km}$. There are typically three RRHs per antenna per mobile radio technology and radio frequency carriers. Three radio technologies (GSM, LTE, LTEa) and radio carriers coexist thus meaning a minimum of 15 ONUs per antenna site.

In this scenario, the RSOA could be placed in the RRH optoelectronic transceivers, the Faraday rotator mirror and the AWG in the antenna cabinet (including power supply, fiber management, local alarm management, etc.), shared by all the ONUs [21]. The cavity length is quite short allowing for longer transmission distances. The great advantage is that instead of using point to point connections for each RRH-BBU link, the selfseeded cavity allows to use only one shared fiber (see Fig. 10) with an automatic and passive wavelength assignment for each ONU.

Recently, a new kind of architecture was demonstrated. The mirror was placed after the demultiplexer, at the receiver side, removing the shared point. To overcome the added losses and improve the data cancellation, a saturated RSOA replaced the Faraday rotator mirror [11], [22]-[24] in a so called "face to face" configuration. Thus, a distance of $70 \mathrm{~km}$ at $2.5 \mathrm{~Gb} / \mathrm{s}$ in the $\mathrm{O}$ band was achieved [24]. With the addition of two SOAs, a $125 \mathrm{~km}$ long cavity was accomplished [11]. By increasing considerably the cavity length, this configuration opens new perspectives. The BBU could be moved directly to the $\mathrm{CO}$ further reducing the infrastructure cost.

\section{CONCLUSION}

By measuring both the light-current curve and the output spectrum of a self-seeded cavity, we have highlighted its lasing behavior: first the threshold occurs when the gain compensates the losses and then, the FSR observed for different cavity lengths matches with the external cavity laser model. Yet, this low coherence laser has to fulfill several conditions to work properly. First, the RSOA should present a sufficient electro-optical response to work at high bit rates; it should erase the returning signal and present a high gain to ensure a satisfying optical budget. Then, a Faraday rotator mirror is mandatory to manage the polarization inside the cavity. A remaining limitation is the noise induced by the longitudinal modes.

The self-seeded cavity architecture is both colorless and passive which fits well for WDM access networks such as the mobile front-haul or enterprise networks. Depending on the application we could either choose the $\mathrm{C}$ band to avoid large fiber losses or the $\mathrm{O}$ band to avoid large chromatic dispersion penalties. Up to now, the possible implementations have shown good performances: the standard architecture reached data rates up to $10 \mathrm{~Gb} / \mathrm{s}$ whereas the "face to face" configuration achieved a transmission distance of $125 \mathrm{~km}$. Both these architectures could be implemented in future standards for self-organized wavelength networks.

\section{REFERENCES}

[1] E. Wong, K. Lee, and T. Anderson, "Directly modulated self-seeding reflective semiconductor optical amplifiers as colourless transmitters in WDM PON," J. Lightw. Technol., vol. 25, no. 1, pp. 67-74, Jan. 2007.

[2] M. Presi and E. Ciaramella, "Stable self-seeding of reflective-SOAs for WDM-PONs," presented at the Optical Fiber Communication Conf./Nat. Fiber Optic Engineers Conf., Los Angeles, CA, USA, 2011, Paper OMP4.

[3] U. R. Duarte, R. S. Penze, F. R. Pereira, F. F. Padela, J. B. Rosolem, and M. A. Romero, "Combined self-seeding and carrier remodulation scheme for WDM-PON," J. Lightw. Technol., vol. 31, no. 8, pp. 1323-1330, Apr. 2013.

[4] C. N. Cheng, Z. X. H. Lin, and D. Liu, "20Gb/s hybrid TDM/WDM PONs with 512-split using self-seeded reflective semiconductor optical amplifiers," in Proc. Opt. Fiber Commun. Conf. Expo. Nat. Fiber Opt. Eng. Conf., Mar. 4-8, 2012, pp. 1-3.

[5] T. Komljenovic, D. Babic, and Z. Sipus, "Modulation-averaging reflectors for extended-cavity optical sources," J. Lightw. Technol., vol. 29, no. 15, pp. 2249-2258, Aug. 2011.

[6] L. Marazzi, P. Parolari, M. Brunero, A. Gatto, M. Martinelli, R. Brenot, S. Barbet, P. Galli, and G. Gavioli, "Up to 10.7-Gb/s high-PDG RSOAbased colorless transmitter for WDM networks," IEEE Photon. Technol. Lett., vol. 25, no. 7, pp. 637-640, Apr. 2013.

[7] P. Parolari, L. Marazzi, M. Brunero, M. Martinelli, R. Brenot, A. Maho, S. Barbet, G. Gavioli, G. Simon, F. Saliou, and P. Chanclou, "10-Gb/s operation of a colorless self-seeded transmitter over more than $70 \mathrm{~km}$ of SSMF," IEEE Photon. Technol. Lett, vol. 26, no. 6, pp. 599-602, Mar. 2014

[8] M. Presi, A. Chiuchiarelli, R. Corsini, and E. Ciaramella, "Uncooled and polarization independent operation of self-seeded Fabry-Pérot lasers for WDM-PONs," IEEE Photon. Technol. Lett., vol. 24, no. 17, pp. 15231526, Sep. 2012.

[9] M. Martinelli, L. Marazzi, P. Parolari, M. Brunero, and G. Gavioli, "Polarization in retracing circuits for WDM-PON," IEEE Photon. Technol. Lett., vol. 24, no. 14, pp. 1191-1193, Jul. 15, 2012.

[10] S. A. Gebrewold, L. Marazzi, P. Parolari, R. Brenot, S. P. O. Dúill, R. Bonjour, D. Hillerkuss, C. Hafner, and J. Leuthold, "Reflective-SOA fiber cavity laser as directly modulated WDM-PON colorless transmitter," IEEE J. Sel. Topics Quantum Electron., vol. 20, no. 5, pp. 503-511, Sep./Oct. 2014

[11] F. Saliou et al., "125-km long cavity based on self-seeded RSOAS colorless sources for 2.5-Gb/s DWDM networks," J. Lightwav. Technol., vol. 33, no. 8, pp. 1602-1607, Apr. 015.

[12] S. O. Duill et al., "Efficient modulation cancellation using reflective SOAs," Opt. Exp., vol. 20, pp. 587-594, 2012. 
[13] P. Parolari et al., "C- and O-band operation of RSOA WDM PON selfseeded transmitters up to $10 \mathrm{~Gb} / \mathrm{s}$ [Invited]," J. Opt. Commun. Netw., vol. 7, pp. A249-A255, 2015.

[14] F. Xiong, W. Zhong, M. Zhu, H. Kim, Z. Xu, and D. Liu, "Characterization of directly modulated self-seeded reflective semiconductor optical amplifiers utilized as colorless transmitters in WDM-PONs," J. Lightw. Technol., vol. 31, no. 11, pp. 1727-1733, Jun. 2013.

[15] M. Brunero, L. Marazzi, P. Parolari, A. Maho, R. Brenot, and M. Martinelli "Experimental evaluation of burst-mode operation of a RSOA-based self-seeded transmitter," presented at the 40th European Optical Communication Conf., Cannes, France, 2014, Paper P7.20.

[16] G. Simon, F. Saliou, P. Chanclou, and R. Brenot, "Infrastructure impact on transmission performances of self seeded DWDM colorless sources at 2.5Gbps," in Proc. Eur. Conf. Opt. Commun., Cannes, France, Sep. 21-25, 2014, pp. 1-3.

[17] P. Parolari, L. Marazzi, M. Brunero, P. Galli, G. Gavioli, and M. Martinelli, "Embedded modulable self-tuning cavity for WDM-PON transmitter" presented at the 14th Int. Conf. Transparent Optical Networks, Coventry, U.K., 2012, Paper Tu.P.4.

[18] Q. Deniel, F. Saliou, P. Chanclou, and D. Erasme, "Self-seeded RSOA based WDM-PON transmission capacities," in Proc. Opt. Fiber Commun. Conf. Expo. Nat. Fiber Opt. Eng. Conf., Anaheim, CA, USA, Mar. 17-21, 2013, pp. 1-3.

[19] S. D. Le, Q. Deniel, F. Saliou, A. Lebreton, and P. Chanclou, " $16 \times 2.5$ $\mathrm{Gb} / \mathrm{s}$ and $5 \mathrm{~Gb} / \mathrm{s}$ WDM PON based on self-seeded RSOA," in Proc. 15th Int. Conf. Transparent Opt. Netw., Cartagena, Colombia, Jun. 23-27, 2013, pp. 1-4.

[20] Y. Ma, D. Liu, J. Yu, and X. Wanf, "System evaluation of economic $16 \times 32$ CHS 1.25 Gbps WDM-PON with self-seeded RSOA," Opt. Exp., vol. 20, pp. 22523-22530, 2012.

[21] P. Parolari et al., "Self-tuning transmitter for fibre-to-the-antenna PON networks," Opt. Switch. Netw., vol. 14, pp. 25-31, 2014.

[22] Q. Deniel, F. Saliou, P. Chanclou, D. Erasme, and R. Brenot, "Up to $45 \mathrm{~km}-$ long amplified self-seeded RSOA based external cavity for $2.5 \mathrm{~Gb} / \mathrm{s}$ WDM PON transmission," presented at the Optical Fiber Communication Conf. Exposition and the Nat. Fiber Optic Engineers Conf., Anaheim, CA, USA, Mar. 17-21, 2013, pp. 1-3.

[23] Q. Deniel, F. Saliou, S. D. Le, P. Chanclou, D. Erasme, and R. Brenot, "Amplified RSOA self-tuning laser for WDM PON using saturated SOA for noise reduction and data cancellation," in Proc. 39th Eur. Conf. Exhib. Opt. Commun., London, U.K., Sep. 22-26, 2013, pp. 1-3.

[24] G. Simon, F. Saliou, P. Chanclou, Q. Deniel, D. Erasme, and R. Brenot, "70 $\mathrm{km}$ external cavity DWDM sources based on O-band self seeded RSOAs for transmissions at 2.5Gbit/s," in Proc. Opt. Fiber Commun. Conf. Exhib., San Francisco, CA, USA, Mar. 9-13, 2014, pp. 1-3.

[25] L. Marazzi, A. Boletti, P. Parolari, A. Gatto, R. Brenot, and M. Martinelli, "Relative intensity noise suppression in reflective SOAs" Opt. Commun., vol. 318, pp. 186-188, 2014.

[26] P. Parolari et al., "Operation of a RSOA WDM PON self-seeded transmitter over more than $50 \mathrm{~km}$ of SSMF up to $10 \mathrm{~Gb} / \mathrm{s}$," presented at the Opt. Fiber Commun. Conf., San Francisco, CA, USA, 2014, Paper W3G.4. 